\title{
Medical abortion in Canada: behind the times
}

\author{
Sheila Dunn MD MSc, Rebecca Cook JD JSD
}

A n estimated 1 in 3 Canadian women will have an abortion during her lifetime, most commonly performed in the first trimester of pregnancy. ${ }^{1}$ However, Canadian women lack access to a safe, effective and often preferred method of early abortion that is available in many other countries. The internationally recognized "gold standard" for medical (i.e., nonsurgical) abortion, mifepristone (followed by misoprostol), is not available in Canada. Although registered in 57 countries, mifepristone has yet to be approved and distributed in this country (Figure 1). Mifepristone became available in France and China in 1988, in the United Kingdom in 1991 and in most European countries by 1999 . It was approved in the United States in 2000 and in Australia in 2012. Mifepristone is included in the WHO Model List of Essential Medicines, ${ }^{2}$ and yet Canadian women do not have access to it.

There is now some hope that the situation may change. In 2012, a pharmaceutical company that supplies mifepristone in many countries, including France, Australia and Sweden, brought forward the first known new drug submission for mifepristone to Health Canada. The results of the submission are pending. It is important that this submission not be allowed to fail.

Medical abortion uses drugs rather than surgery to induce an early abortion. Mifepristone, developed in the 1980s for medical abortion, is an orally administered antiprogestin that blocks the action of progesterone, causing degeneration of the endometrial lining, softening of the cervix and sensitization to prostaglandins. Administration of a prostaglandin, typically the widely available misoprostol, at home 1-2 days after administration of mifepristone causes expulsion of the destabilized products of conception in a process similar to early miscarriage. ${ }^{2}$

Millions of women worldwide have used mifepristone safely and effectively. A recent systematic review of 45000 abortions with mifepristone found that ongoing pregnancy occurred in only $1.1 \%$ of patients, less than $5 \%$ received a surgical procedure to complete the abortion and serious complications occurred in only $0.4 \%$. $^{3}$ Abortion with mifepristone is increasingly common and now accounts for more than $60 \%$ of abortions in some European countries and about $20 \%$ of abortions in the US. ${ }^{3,4}$

Unfortunately, Canadian women who want a medical abortion (assuming they can find a provider) must resort to a more cumbersome method that uses the cytotoxic drug methotrexate, followed 5-7 days later by misoprostol. Although the methotrexate-misoprostol approach provides a nonsurgical option, it is the second-best method. If allowed to take its course, the regimen is as effective as mifepristone and misoprostol for abortions at up to 7 weeks' gestation, but its time course is longer and less predictable, with some abortions delayed several weeks after administration of methotrexate. ${ }^{5}$ Because methotrexate is teratogenic, the World Health Organization does not recommend it for abortion because of its association with serious deformities in the infant if the abortion fails and the pregnancy continues. ${ }^{2}$

In a randomized trial comparing the 2 methods, women found mifepristone to be more acceptable than methotrexate overall ( $88 \%$ v. $83 \%, p<0.03)$, but more so for pain $(86 \%$ v. $78 \%, p<0.001)$ and wait time $(92 \%$ v. $80 \%, p<0.001) .{ }^{5}$ Abortions with methotrexate are also labour-intensive for practitioners, who must be willing to obtain the drug, administer it intramuscularly and follow women over weeks to ensure the abortion is complete. Therefore, it is not surprising that medical abortion is not widely used in Canada. A recent study from British Columbia, where most medical abortions in Canada are thought to be performed by a few large-volume providers, showed that $15 \%$ of abortions are done medically. ${ }^{6}$ Rates are thought to be much lower elsewhere in Canada.

In fact, access to any type of abortion remains limited in many areas across Canada. A recent
Competing interests: Sheila Dunn is on the board of the National Abortion Federation, a nonprofit agency that promotes access to quality abortion care in the United States, Canada and Latin America. No competing interests were declared by Rebecca Cook.

This article was solicited and has not been peer reviewed.

Correspondence to:

Sheila Dunn,

sheila.dunn@wchospital.ca

CMAJ 2014. DOI:10.1503 /cmaj.131320 


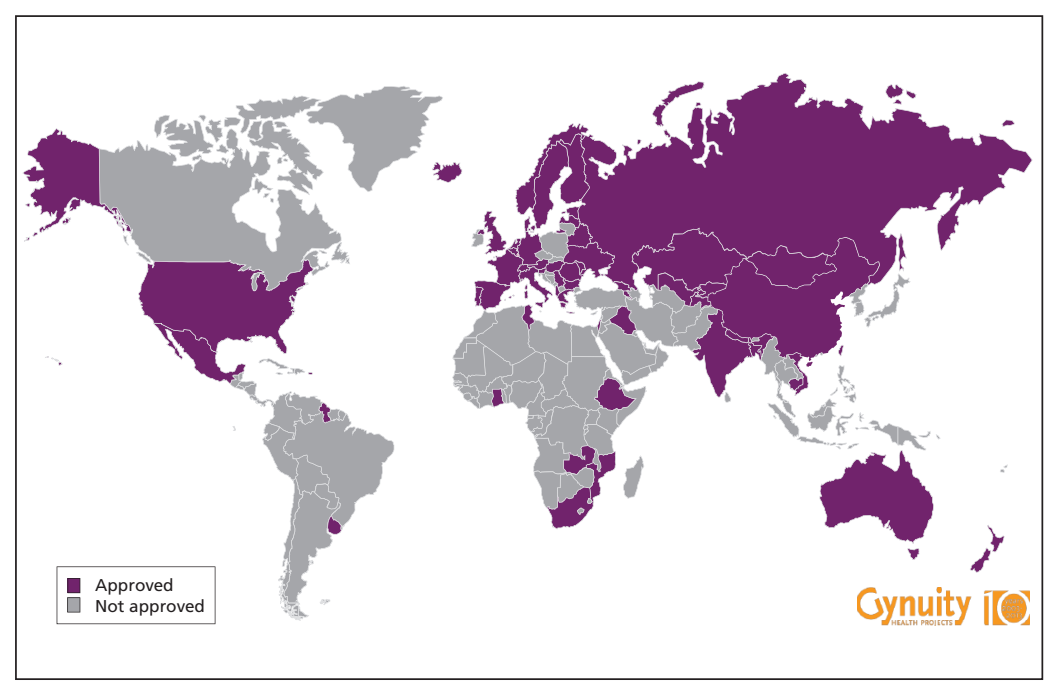

Figure 1: Approval of mifepristone between 1988 and 2013. Reproduced with permission. @2013 Gynuity Health Projects.

study that mapped women's travel to abortion clinics showed that women from Atlantic Canada and those from rural and northern communities often travel long distances and face long wait times to obtain services. ${ }^{7}$ Better access to mifepristone abortion provided by primary care providers, including family physicians, nurse practitioners and midwives, could reduce these gaps in service delivery and free up operating room time. Availability of mifepristone in other countries has been associated with abortions occurring earlier in gestation, when they are safer, and in reduced wait times for surgical abortions. ${ }^{4}$

In 2009, The Society of Obstetricians and Gynaecologists of Canada urged Health Canada "to work with professional organizations and industry to make this product (mifepristone) available to women living in Canada." ${ }^{8}$ So why is mifepristone still not available in Canada? One factor may be that pharmaceutical companies are deterred from bringing forward submissions because the Canadian market is relatively small, and obtaining regulatory approval can be costly. Some have also argued that Health Canada is biased against reproductive health medications, with more onerous requirements for regulatory approval compared with Europe and the US. ${ }^{9,10}$ Drug approval generally takes longer in Canada than in the US, but the lag time difference is longer for contraceptives. ${ }^{10}$

Is the Canadian government shirking its responsibility to facilitate availability of mifepristone, as some have suggested? ${ }^{9}$ Health Canada is accountable under the Canadian Charter of Rights and Freedoms to not unreasonably obstruct or delay women's exercise of their rights to security, liberty and equality. Failure to provide essential drugs that only women need, including mifepristone, is a form of discrimination that Canada is obligated to remedy. Moreover, Canada, as a party to the International Covenant on Economic, Social and Cultural Rights, has a responsibility to undertake legislative, administrative and budgetary measures to ensure women's right to health through access to essential reproductive health medicines.'

It is time that Canadian women had the ability to choose the best regimen for medical abortion. Availability of mifepristone, with the attendant probability of reduced demand and therefore shortened wait lists for surgical abortions, could improve the capacity of the health care system to provide abortions earlier, when they are safest. Moreover, the distribution of mifepristone would enhance access to abortion, particularly among underserved populations. Ultimately, the availability of mifepristone in Canada would provide an important therapy that would help to optimize the health of Canadian women.

\section{References}

1. Norman WV. Induced abortion in Canada 1974-2005: trends over the first generation with legal access. Contraception 2012; 85:185-91.

2. Safe abortion: technical and policy guidance for health systems. 2nd ed. Geneva: World Health Organization; 2012. Available: http://apps.who.int/iris/bitstream/10665/70914/1/978924154843 4_eng.pdf (accessed 2013 Aug. 21).

3. Raymond EG, Shannon C, Weaver MA, et al. First-trimester medical abortion with mifepristone $200 \mathrm{mg}$ and misoprostol: a systematic review. Contraception 2013;87:26-37.

4. Jones RK, Henshaw S. Mifepristone for early medical abortion: experiences in France, Great Britain and Sweden. Perspect Sex Reprod Health 2002;34:154-61.

5. Wiebe E, Dunn S, Guilbert E, et al. Comparison of abortions induced by methotrexate or mifepristone followed by misoprostol. Obstet Gynecol 2002;99:813-9.

6. Norman WV, Soon JA, Maughn N, et al. Barriers to rural induced abortion services in Canada: findings of the British Columbia Abortion Providers Survey (BCAPS). PLOS ONE 2013;8:e67023.

7. Sethna C, Doull M. Spatial disparities and travel to freestanding abortion clinics in Canada. Womens Stud Int Forum 2013;38:52-62.

8. Executive Committee of the Society of Obstetricians and Gynaecologists of Canada (SOGC). Mifepristone. J Obstet Gynaecol Can 2009;31:1180-1.

9. Erdman JN, Grenon A, Harrison-Wilson L. Medication abortion in Canada: a right-to-health perspective. Am J Public Health 2008; 98:1764-9.

10. Azzarello D, Collins J. Canadian access to hormonal contraceptive drug choices. J Obstet Gynaecol Can 2004;26:489-500.

Affiliations: Department of Family and Community Medicine (Dunn), Women's College Hospital, Toronto, Ont.; Faculty of Law (Cook), University of Toronto, Toronto, Ont.

Contributors: Both authors developed the idea for the commentary. Sheila Dunn wrote the medical content, Rebecca Cook wrote the legal content and both authors contributed to the policy component. Both authors approved the final version submitted for publication. 